\title{
Serum lipoproteins and apolipoproteins in young normocholesterolaemic, non-diabetic Indian men with myocardial infarction
}

\author{
M. Sewdarsen', R.K. Desai ${ }^{2}$, S. Vythilingum ${ }^{1}$, N. Shah ${ }^{2}$ and M.C. Rajput ${ }^{1}$ \\ ${ }^{1}$ Ischaemic Heart Disease Clinic, Department of Medicine, R.K. Khan Hospital, and ${ }^{2}$ Department of \\ Chemical Pathology, University of Natal Medical School, Durban, Natal, South Africa
}

Summary: Serum total cholesterol, triglycerides, high density lipoprotein cholesterol, low density lipoprotein cholesterol, apolipoprotein A-I and apolipoprotein B were evaluated as potential indicators of the risk of coronary artery disease in young $(<46$ years) normocholesterolaemic, non-diabetic men who had previously sustained a myocardial infarction $(n=50)$ and in healthy age and sex matched controls $(n=122)$ with a similar socioeconomic background.

Significant differences were observed between patients and controls in the mean concentrations of serum total cholesterol, triglycerides, low density lipoprotein cholesterol, high density lipoprotein cholesterol and apolipoprotein B, as well as in the ratios of total cholesterol to high density lipoprotein cholesterol and apolipoprotein A-I to apolipoprotein B. No significant difference was demonstrated in the concentration of apolipoprotein A-I between the two groups. Stepwise discriminant analysis indicated that apolipoprotein B was the best discriminant between patients and controls. The percentage of exact classification was $74 \%$ in patients and $66 \%$ in controls.

When the patients were compared to a subset of controls $(n=50)$ matched for age and total cholesterol, significant differences were demonstrated only in the mean concentrations of apolipoprotein $B$. Discriminant analysis confirmed that the best single discriminating variable was apolipoprotein $B$.

The results therefore indicate that in young normocholesterolaemic, non-diabetic Indian men with myocardial infarction, apolipoprotein B is superior to other lipid parameters studied, as a marker for coronary artery disease.

\section{Introduction}

Risk factors for the development of myocardial infarction, particularly lipid abnormalities, have been considered to be more pronounced in young patients than in middle aged or elderly individuals. ${ }^{1,2}$ However, the risk of coronary artery disease is only partially characterized by lipid abnormalities since there are many individuals who develop the disease but have normal serum lipid and lipoprotein cholesterol levels. ${ }^{3}$ Though the disease may be related to the presence of other risk factors such as hypertension, diabetes mellitus and cigarette smoking in some of these individuals, the presence of coronary artery disease in a significant number of patients remains unexplained. Moreover, age-dependent degenerative disease in young patients with coronary artery disease may be considered minimal. Hence interest has focused on measurements of apolipoproteins because of their pivotal roles in cholesterol transport and metabolism. ${ }^{4}$

Correspondence: M. Sewdarsen, M.B., Ch.B.

Accepted: 16 August 1990
Associations between apolipoprotein B (apo B) and apolipoprotein A-I (apo A-I) concentrations and coronary artery disease have been confirmed in several case control studies. ${ }^{5-8}$ Opinions are divided, however, as to whether increases in apo B, or decreases in apo A-I, are better indicators of coronary artery disease risk than total cholesterol, low density lipoprotein (LDL) and high density lipoprotein (HDL) cholesterol. ${ }^{9-11}$

Recent studies which have addressed this question have produced conflicting results, ${ }^{9-12}$ though the majority of studies have suggested that concentrations of apolipoproteins, in particular apo B and possibly also apo A-I, discriminate better than cholesterol and lipoprotein concentrations between patients with coronary artery disease and controls.

Accordingly the present investigation focuses on serum apo A-I and apo B in young Indian survivors of myocardial infarction and healthy age and sex matched controls. The object of the study was to investigate the importance of apolipoproteins as risk factors in a sample of young normocholestero- 
laemic and non-diabetic Indian males with myocardial infarction.

\section{Patients and methods}

Male patients below the age of 46 years attending the Ischaemic Heart Disease Clinic at the R.K. Khan Hospital and who fulfilled the following prerequisites were considered for this study: (1) admission to the coronary care unit at R.K. Khan Hospital with documented myocardial infarction at least 6 months previously. The diagnosis of myocardial infarction was based on a typical history of central chest pain, accompanied by unequivocal electrocardiographic changes of acute myocardial infarction and transient rise in creatine kinase levels. (2) Normal glucose tolerance test and total serum cholesterol $\leqslant 6.5 \mathrm{mmol} / \mathrm{l}$. The cut-off point for total cholesterol was chosen in order to exclude patients with high values, thus permitting an investigation of normocholesterolaemic, nondiabetic patients. These tests were performed routinely 3 months after the acute episode of myocardial infarction. (3) Stable clinical condition. Patients on diuretics were excluded whilst treatment with beta blockers and calcium channel blockers was not a criterion for exclusion.

Consecutive patients attending the clinic and who fulfilled these criteria were recalled for repeat cholesterol, triglyceride, lipoprotein and apolipoprotein analyses. Any patient in whom the repeat metabolic evaluation revealed total cholesterol $>6.5 \mathrm{mmol} / \mathrm{l}$ was excluded. The first 50 consecutive patients who satisfied all the criteria were selected. The study group hence consisted of 50 non-diabetic patients between the ages of 26 and 45 years with total cholesterol $\leqslant 6.5 \mathrm{mmol} / 1$ on two separate occasions. Of these patients, 2 were on calcium channel blockers and 19 on beta blockers (16 on atenolol and 3 on acebutalol). No patients had been on lipid lowering drugs. The patients were studied at a mean of 17 months (range 6-36 months) after the myocardial infarction. Eight patients were hypertensive.

As controls, 122 normocholesterolaemic male volunteers, employed at the hospital, with no personal history of coronary artery disease or diabetes, were studied. The clinical examination in all controls was normal. Electrocardiograms were not done in the control population. The socioeconomic status of the controls was similar to the patients.

The patients and controls were investigated simultaneously. Venous blood was obtained in all participants after a 14 hour fast between $08.00 \mathrm{~h}$ and $10.00 \mathrm{~h}$. Height and weight were recorded in all individuals and body mass index calculated by the formula weight $(\mathrm{kg}) / \mathrm{height}(\mathrm{m})^{2}$. Other historical data obtained from all participants included smoking habits and family history of coronary artery disease. The family history was considered positive if there was a history of myocardial infarction before age 60 years in parents or siblings.

Measurements of serum total cholesterol, trigly cerides, high density lipoprotein (HDL) cholesterol and low density lipoprotein (LDL) cholesterol were performed as described previously. ${ }^{13,14}$ Briefly total cholesterol and triglycerides were measured by enzymatic methods utilizing commercial kits (Boehringer Mannheim); HDL-cholesterol was measured by a polyanion precipitation method using phosphotungstate and magnesium chloride and LDL-cholesterol by polyvinyl sulphate precipitation method (Boehringer Mannheim). The apolipoprotein measurements were made by rate nephelometry ${ }^{15}$ with reagents supplied by Beckman (Beckman Instruments Inc, California). This test measures the rate of increase in light scattered from particles suspended in solution as a result of complexes formed during an antigen-antibody reaction. The values for apo A-I and apo B obtained by this method correlate satisfactorily with values obtained by radial immunodiffusion (correlation coefficient $=0.93$ for apo A-I and 0.96 for apo B). ${ }^{15}$ The intra- and inter-assay coefficients of variation for both apo A-I and apo B determinae tions were $\leqslant 5 \%$.

The importance of apolipoprotein in survivors was further evaluated by a comparison with $\dot{a}$ subset of controls that were matched for cholesterol with the myocardial infarct survivors.

\section{Statistical analyses}

Proportions were compared using the chi-square test with Yates' correction. Statistical significance for differences in continuous variables between groups was tested using one way analysis of variance and two-tailed $t$-test. Triglyceride values were $\log$ transformed before statistical analysis but untransformed values are given in the text and tables. Stepwise multiple discriminant analyses were performed to determine the sets of independent lipoprotein and apolipoprotein variables giving the best discrimination between survivors and controls. The statistical analyses were performed with BMDP statistical software (University of California Press, Berkeley, California). All data in text and tables are means and 1 standard deviation (s.d.).

\section{Ethical consideration}

Prior to the study informed consent was obtained from all subjects. The study protocol had been approved by the hospital administration. 


\section{Results}

Clinical data for the patient and control groups are listed in Table I. At the time of the metabolic evaluation the smoking histories of the patients were similar to the controls. However there was a significant larger number of ex-smokers in the patient group, the majority of whom had stopped smoking at the time of the myocardial infarction. Although a history of myocardial infarction in first degree relatives was encountered more frequently in the patients, the difference was not statistically significant.

Men with myocardial infarction had significantly higher levels of serum total cholesterol $(P<$ $0.001)$, triglycerides $(P<0.005)$, LDL-cholesterol $(P<0.005)$ and apo B $(P<0.0001)$ and significantly lower levels of HDL-cholesterol $(P<0.01)$ than healthy age matched controls (Table II). Apolipoprotein A-I levels did not differ between patients and controls. Significantly higher total cholesterol/HDL-cholesterol and lower apo A-I/ apo $B$ ratios were noted in patients when compared to controls (Table II). Table III shows the proportion of patients with abnormal values for total cholesterol, triglycerides, lipoprotein and apolipoprotein fractions. For serum cholesterol, triglyceride, LDL-cholesterol and apo B, concentrations above the 90th percentile, and for HDL-cholesterol and apo A-I, values below the 10th percentile of the results obtained in controls were used to define abnormality. The highest percent of abnormal values was noted for apo B concentrations $(36 \%$, 95\% CI $22-50 \%$ ).

Stepwise discriminant analyses were performed to determine which variables were most important in differentiating between men with myocardial infarction and those without. Variables included in the analysis included body mass index, family history of myocardial infarction, total cholesterol, triglycerides, HDL-cholesterol, LDL-cholesterol, apo A-I, apo B and the ratios of total cholesterol to HDL-cholesterol and apo A-I to apo B. The first

Table I Characteristics of patients with myocardial infarction and healthy controls

\begin{tabular}{lccc}
\hline & Patients & Controls & P value \\
\hline$n$ & 50 & 122 & \\
Age (years) & $39 \pm 5$ & $37 \pm 6$ & NS \\
Body mass index & $24.6 \pm 3.4$ & $23.6 \pm 3.2$ & NS \\
Cigarette smokers & $19(38)$ & $56(46)$ & NS \\
$\quad[n(\%)]$ & & & \\
Former smokers $[n(\%)]$ & $21(42)$ & $12(10)$ & $<0.0001$ \\
F-CAD $[n(\%)]$ & $22(44)$ & $35(29)$ & NS \\
\hline
\end{tabular}

Values are mean \pm 1 s.d. except where indicated. F-CAD $=$ family history of premature myocardial infarction in first degree relatives.
Table II Mean ( \pm 1 s.d.) concentrations of serum lipids $(\mathrm{mmol} / \mathrm{l})$, lipoproteins $(\mathrm{mmol} / \mathrm{l})$ and apolipoproteins $(\mathrm{g} / \mathrm{l})$ in men with myocardial infarction and controls

\begin{tabular}{lcccc}
\hline & $\begin{array}{c}\text { Patients } \\
(n=50)\end{array}$ & $\begin{array}{c}\text { Controls } \\
(n=122)\end{array}$ & P value \\
\hline Total cholesterol & $5.9 \pm 0.8$ & $5.4 \pm 0.8$ & $<0.001$ \\
Triglycerides* & $2.2 \pm 1.4$ & $1.7 \pm 1.0$ & $<0.005$ \\
HDL-cholesterol & $0.8 \pm 0.2$ & $0.9 \pm 0.2$ & $<0.01$ \\
LDL-cholesterol & $4.0 \pm 0.9$ & $3.6 \pm 0.8$ & $<0.005$ \\
Apolipoprotein A-I & $1.1 \pm 0.2$ & $1.2 \pm 0.3$ & $\mathrm{NS}$ \\
Apolipoprotein B & $1.1 \pm 0.2$ & $0.9 \pm 0.2$ & $<0.0001$ \\
Cholesterol/HDL ${ }^{\dagger}$ & $7.3 \pm 1.9$ & $6.1 \pm 1.7$ & $<0.0001$ \\
Apo A/Apo B & $1.1 \pm 0.3$ & $1.4 \pm 0.4$ & $<0.0005$
\end{tabular}

HDL - high density lipoprotein; LDL - low density lipoprotein; Apo A-I = apolipoprotein A-I; Apo B = apolipoprotein B; ${ }^{*}$ Geometric mean (range): patients 1.9 (7.1) $\mathrm{mmol} / \mathrm{l}$; controls 1.5 (5.8) $\mathrm{mmol} / \mathrm{l}$; ${ }^{\dagger}$ Ratio of total cholesterol to HDL-cholesterol; ${ }^{\ddagger}$ Ratio of apo A-I to apo B.

Table III Proportions of abnormal values in normocholesterolaemic patients with myocardial infarction

\begin{tabular}{lcr}
\hline & Percent & \multicolumn{1}{c}{$95 \mathrm{CI}$} \\
\hline Total cholesterol $>6.4$ & 26 & $14-38$ \\
HDL-cholesterol $<0.66 \mathrm{mmol} / 1$ & 20 & $8-32$ \\
LDL-cholesterol $>4.6 \mathrm{mmol} / \mathrm{l}$ & 26 & $14-38$ \\
Triglycerides $>3.3 \mathrm{mmol} / 1$ & 12 & $2-22$ \\
Apolipoprotein A-I $<0.9 \mathrm{~g} / \mathrm{l}$ & 16 & $6-26$ \\
Apolipoprotein B $>1.1 \mathrm{~g} / \mathrm{l}$ & 36 & $22-50$
\end{tabular}

For all variables the 90th percentile except for HDLcholesterol and apolipoprotein A-I the 10th percentile values of the controls were used to define abnormality. $\mathrm{CI}=$ confidence interval; $\mathrm{HDL}=$ high density lipoprotein; $\mathrm{LDL}=$ low density lipoprotein.

variable selected was apo B. However once apo B was entered, no other individual variable provided significant additional contribution. With apo $B$ as the only discriminative variable, $66 \%$ of controls and $74 \%$ of patients were correctly identified. The percentage of correctly classified subjects could not be increased by the addition of other variables. The discriminant analysis was then repeated in subjects with and without family history of myocardial infarction without altering the results.

The importance of apoproteins in relation to myocardial infarction was further evaluated by comparison of patients with cholesterol-matched subgroup of controls. The results are presented in Tables IV and V. The data show that of all the variables studied, only apo B values were significantly different in patients with myocardial infarction compared to controls (Table IV). Serum apo B $>1.14$ (90th percentile) was found in $32 \%$ of 
patients with myocardial infarction (Table V). From Table $\mathrm{V}$ it can also be seen that the separation between patients and controls was greatest for apo B, there being a 3.2-fold difference for apo B compared to a 1.6-fold difference for apo A-I in the observed/expected ratios of the two groups.

There was no significant difference in the mean serum lipid, lipoprotein and apolipoprotein values in patients on beta blockers $(n=19)$ when compared to patients not on these drugs (data not presented).

\section{Discussion}

In this study of young, normocholesterolaemic, non-diabetic Indian men, we found that apo B levels were better markers for myocardial infarction than lipid and lipoprotein measurements. The main finding in this study is, in general, consistent with previous work by other investigators. ${ }^{5-7,9,11,12}$

Table IV Selected risk factors in patients with myocardial infarction and in cholesterol-matched controls

\begin{tabular}{lccc}
\hline & $\begin{array}{c}\text { Patients } \\
(n=50)\end{array}$ & $\begin{array}{c}\text { Controls } \\
(n=50)\end{array}$ & $\begin{array}{c}\text { P } \\
\text { value }\end{array}$ \\
\hline Age (years) & $39 \pm 5$ & $39 \pm 5$ & $\mathrm{NS}$ \\
Body mass index & $24.6 \pm 3.4$ & $24.0 \pm 3.2$ & $\mathrm{NS}$ \\
Total cholesterol (mmol/l) & $5.9 \pm 0.8$ & $5.8 \pm 0.7$ & $\mathrm{NS}$ \\
Triglycerides (mmol/l) & $2.2 \pm 1.4$ & $1.9 \pm 1.1$ & $\mathrm{NS}$ \\
HDL-cholesterol (mmol/l) & $0.8 \pm 0.2$ & $0.9 \pm 0.2$ & $\mathrm{NS}$ \\
LDL-cholesterol (mmol/l) & $4.0 \pm 0.9$ & $3.9 \pm 0.7$ & $\mathrm{NS}$ \\
Apo A-I (g/l) & $1.1 \pm 0.2$ & $1.1 \pm 0.2$ & $\mathrm{NS}$ \\
Apo B (g/l) & $1.1 \pm 0.2$ & $0.9 \pm 0.2$ & 0.02 \\
Cholesterol/ & $7.3 \pm 1.9$ & $6.8 \pm 1.8$ & $\mathrm{NS}$ \\
$\quad$ HDL-cholesterol & $1.1 \pm 0.3$ & $1.2 \pm 0.4$ & $\mathrm{NS}$ \\
Apo A-I/Apo B & $22(44)$ & $18(36)$ & $\mathrm{NS}$ \\
F-CAD n(\%) & & \\
\hline
\end{tabular}

All values except where indicated are mean \pm s.d.; HDL $=$ high density lipoprotein; $\mathrm{LDL}=$ low density lipoprotein; Apo A-I = apolipoprotein A-I; Apo B = apolipoprotein $\mathrm{B} ; \mathrm{F}-\mathrm{CAD}=$ family history of myocardial infarction.
In this study, although subjects with total cholesterol below $6.6 \mathrm{mmol} / 1$ were selected, the mean cholesterol of the patients was significantly higher than the controls. In addition significant differences were demonstrated in the mean values of LDL-cholesterol, HDL-cholesterol, triglycerides, apo $B$ and in the ratios of total cholesterol/HDLcholesterol and apo A-I/apo B by univariate analysis, whilst apo A-I levels between the groups were similar. However, stepwise multiple discriminant analysis indicated that apo B had the best discriminative power between survivors and controls. The percentage success for exact classification using apo B was $74 \%$ for patients and $66 \%$ for controls. Neither the inclusion of other lipoprotein or lipid concentrations nor the ratios of total cholesterol/ HDL-cholesterol or apo A-I/apo B improved the discrimination provided by apo $B$.

The results of the multivariate analysis concur with several recent reports which have demonstrated that apo B concentrations may be considerably better markers for coronary artery disease than traditional lipid determinations. ${ }^{6,7,9,11}$ However, unlike many of the previous studies, apo B in the present study was the only variable selected in the stepwise procedure and the inclusion of other variables did not improve the discrimination provided by apo $B$.

Our results with respect to apo B indicate that a screening policy for normocholesterolaemic Indian individuals at risk for coronary artery disease is likely to be unsound unless apo B is included. The importance of apo $B$ can be appreciated further by the significant differences in its mean concentrations observed when the patients were compared to cholesterol-matched controls whilst no differences were noted in the other parameters that were measured. In addition, $32 \%$ of the patients had apo B levels above the 90th percentile of the cholesterol-matched controls. In this subanalysis, apo $B$ was again the most discriminative and the only variable selected. This observation gives further support to the recently delineated condition of

Table V Proportions of abnormal values in patients and cholesterolmatched controls

\begin{tabular}{lcccc}
\hline & $\begin{array}{c}\text { Value } \\
(\text { mmol/l) }\end{array}$ & Expected $^{*}$ & Observed $^{\dagger}$ & $\begin{array}{c}\text { Observed/ } \\
\text { Expected }^{-}\end{array}$ \\
\hline LDL-cholesterol & $>4.8$ & 10 & 24 & 2.4 \\
Triglycerides & $>3.8$ & 10 & 6 & 0.6 \\
HDL-cholesterol & $<0.65$ & 10 & 16 & 1.6 \\
Apolipoprotein A-I & $<0.93$ & 10 & 16 & 1.6 \\
Apolipoprotein B & $>1.14$ & 10 & 32 & 3.2 \\
\hline
\end{tabular}

For all variables the 90th percentile except for HDL-cholesterol and apolipoprotein A-I the 10th percentile of the controls were used to define abnormality. "Percent of controls with abnormal levels; ${ }^{\dagger}$ percent of patients with abnormal levels. 
hyperapobetalipoproteinaemia in normolipidaemic patients. ${ }^{16,17}$

HDL data emerging from this study showed that as a single variable HDL-cholesterol provided a better means of discrimination between patients and controls than did apo A-I, the major apolipoprotein of HDL-cholesterol. Though many researchers have demonstrated significant reduced levels of apo A-I in subjects with coronary artery disease, ${ }^{6-9,12}$ cases reporting low HDL-cholesterol with normal apo A-I values have been documented. ${ }^{10}$

There is much debate on whether serum triglyceride is a risk factor for coronary artery disease and this issue remains unresolved. ${ }^{18}$ In our study, whilst there was a significant difference in serum triglyceride concentrations between patients and controls by univariate analysis, multivariate analysis failed to select this variable as a significant factor.

The findings in this study should be interpreted with due regard to its cross-sectional design and selection of patients. Significant changes in the associations between lipids and myocardial infarction may occur during the time between the onset of the disease and the metabolic evaluation as a result of diet modification and change of life style. However, such alterations are less probable in these patients since their total cholesterol levels were normal; they were generally well following their infarction, had all returned to work and were in a stable clinical condition at the time of the study. Sufficient time was allowed between myocardial infarction and the study to reverse the temporary effects of acute phasic reactions on cholesterol, triglycerides, lipoproteins and apolipoproteins. ${ }^{19,20}$

\section{References}

1. Bergstrand, R., Vedin, A., Wilhelmsson, C., Wallin, J., Wedel, H. \& Wilhelmsen, C. Myocardial infarction among men below age 40. Br Heart $J$ 1978, 40: 783-788.

2. Kannel, W.B., Garcia, M.J., McNamara, P.M. \& Pearson, G. Serum lipid precursors of coronary heart disease. Hum Pathol 1971, 2: 129-151.

3. Holmes, D.R., Elveback, L.R., Frye, R.C., Kottke, B.A. \& Ellefson, R.D. Association of risk factor variables and coronary artery disease documented with angiography. Circulation 1981, 63: 293-299.

4. Thompson, G. Apoproteins: determinants of lipoprotein metabolism and indices of coronary risk. Br Heart J 1984, 51 : $585-588$.

5. De Backer, G., Rosseneu, M. \& Deslypere, J.P. Discriminative value of lipid and apoproteins in coronary heart disease. Atherosclerosis 1982, 42: 197-203.

6. Avogaro, P., Bittolo Bon, G., Cazzolato, G. \& Rorai, E. Relationship between apolipoproteins and chemical components of lipoproteins in survivors of myocardial infarction. Atherosclerosis 1980, 37: 69-76.

7. Riesen, W.F., Mordasini, R., Salzmann, C., Theler, A. \& Gurtner, H.P. Apoproteins and lipids as discriminators of severity of coronary heart disease. Atherosclerosis 1980, 37: $157-162$.
Since no difference in the lipid profile was observed between patients with or without beta blockers, and patients on diuretics were excluded, medication cannot account for the differences observed between the patients and controls. Apolipoprotein abnormalities have been demonstrated in relatives of patients with coronary artery disease. ${ }^{21}$ It is unlikely that familial history of myocardial infarction influenced our findings. Despite a high frequency of positive family history in both patients and controls, the difference was not statistically significant and, furthermore, discriminant analysis performed separately in subjects with and without a positive history yielded identical results.

Our study by virtue of its cross-sectional design does not allow conclusions to be drawn about the predictive value of the various lipid, lipoprotein and apolipoprotein levels. Such a consideration will have to await results from prospective studies. Nevertheless the results of the present study indicate that apo B levels are superior to lipoprotein determinations as a marker for coronary artery disease in young non-diabetic, normocholesterolaemic patients with myocardial infarction.

\section{Acknowledgements}

We thank Mr P. Ganputh for the biochemical analyses, the nurses of the coronary care unit and the ischaemic heart disease clinic for their co-operation, Dr P.J. Becker and Ms Esther Viljoen, of the Institute for Biostatistics of the South African Medical Research Council, for undertaking the statistical analyses, and Dr P.K. Naidoo, Medical Superintendent of R.K. Khan Hospital, for permission to publish our findings.

8. Onitiri, A.C. \& Jover, E. Comparative serum apolipoprotein studies in ischaemic heart disease and control subjects. Clin Chim Acta 1980, 108: 25-30.

9. Durrington, P.N., Hunt, L., Ishola, M., Kane, J. \& Stephens, W.P. Serum apolipoprotein AI and B and lipoproteins in middle aged men with and without previous myocardial infarction. Br Heart J 1986, 56: 206-212.

10. Schmidt, S.B., Wassermann, A.G., Muesing, R.A., Schlesselman, S.E., Larosa, J.C. \& Ross, A.M. Lipoprotein and apolipoprotein levels in angiographically defined coronary atherosclerosis. Am J Cardiol 1985, 55: 1459-1462.

11. Kottke, B.A., Zinsmeister, A.R., Holmes, D.R., Kneller, R.W., Hallaway, B.J. \& Mao, S.J.T. Apolipoproteins and coronary artery disease. Mayo Clin Proc 1986, 61: 313-320.

12. Hamsten, A., Walldius, G., Stamosi, A., Dahlen, G. \& de Faire, U. Relationship of angiographically defined coronary artery disease to serum lipoproteins and apolipoproteins in young survivors of myocardial infarction. Circulation 1986, 73: $1097-1110$.

13. Sewdarsen, M., Jialal, I. \& Vythilingum, S. Abnormal glucose tolerance and lipid abnormalities in Indian myocardial infarct survivors. $S$ Afr Med J 1983, 63: 238-240. 
14. Jialal, I., Deppe, W. \& Joubert, S.M. Lipid aberrations in Indian patients with non insulin dependent diabetes in the young. S Afr Med J 1985, 67: 1001-1004.

15. Sternberg, J.C. A rate nephelometer for measuring specific proteins by immunoprecipitin reactionș. Clin Chem 1977, 23: 1456-1464.

16. Sniderman, A., Shapiro, S., Marpole, D., Skinner, B., Teng, B., Kwiterovich, P.O. Jr. Association of coronary atherosclerosis with hyperapobetalipoproteinemia (increased protein but normal cholesterol levels in human plasma low density ( $\beta$ ) lipoproteins). Proc Natl Acad Sci USA 1980, 77: 604-608.

17. Avogaro, P., Bittolo Bon, G., Cazzolato, G. \& Quinci, G.B. Are apolipoproteins better discriminators than lipids for atherosclerosis? Lancet 1979, i: 901-903.
18. Hulley, S.B., Rosenman, R.H., Bawol, R.D. \& Brand, R.J. Epidemiology as a guide to clinical decisions. The association between triglycerides and coronary heart disease. $N$ Engl $J$ Med 1980, 302: 1383-1389.

19. Avogaro, P., Bittolo Bon, G., Cazzolato, G. et al. Variations in apolipoproteins $B$ and AI during the course of myocardial infarction. Eur J Clin Invest 1978, 8: 121-129.

20. Sewdarsen, M., Vythilingum, S., Jialal, I. \& Nadar, R. Plasma lipids can be reliably assessed within 24 hours after acute myocardial infarction. Postgrad Med J 1988, 64: 352-356.

21. Kukita, H., Hiwada, K. \& Kokubo, T. Serum apolipoprotein A-I, A-II and B levels and their discriminative values in relatives of patients with coronary artery disease. Atherosclerosis 1984, 51: 261-267. 